\title{
Does the addition of donkey milk inhibit the replication of pathogen microorganisms in goat milk at refrigerated condition?
}

\author{
Filippo Fratini ${ }^{1,2} \cdot$ Barbara Turchi $^{1}$ - Francesca Pedonese ${ }^{1,2}$. \\ Federica Pizzurro ${ }^{1}$ - Paola Ragaglini ${ }^{1}$ - Beatrice Torracca ${ }^{1}$. \\ Beatrice Tozzi $^{1} \cdot$ Alessia Galiero $^{1} \cdot$ Roberta Nuvoloni $^{1,2}$
}

\begin{abstract}
Currently, donkey milk is receiving an increasing attention from consumers and research community because of its several beneficial aspects, such as a poor allergenic nature and a remarkable antimicrobial compound content. In this study, we evaluated the growth rate of Staphylococcus aureus ATCC 6538, Listeria monocytogenes ATCC 7644TM, Campylobacter jejuni ATCC 33291, and Pseudomonas aeruginosa ATCC 27853 at refrigeration conditions $\left(4 \pm 2{ }^{\circ} \mathrm{C}\right)$ in goat milk added with different percentages of donkey milk $(1,2.5,5,10 \% v / v)$ along 6 days of storage; furthermore, donkey milk and goat milk samples were employed as controls. Lysozyme content of donkey milk was determined and ranged from $1 \mathrm{mg} \cdot \mathrm{mL}^{-1}$ up to $2 \mathrm{mg} \cdot \mathrm{mL}^{-1}$. An inhibited growth rate in donkey milk samples was observed during storage for S. aureus, L. monocytogenes, and C. jejuni, with a growth decrease of 0.61 $\log$ CFU.mL ${ }^{-1}$, $5.55 \log$ CFU.mL ${ }^{-1}$, and $1.72 \log$ CFU.mL ${ }^{-1}$, respectively. These data confirm the antibacterial activity of donkey milk against Gram-positive and Gramnegative microorganisms; however, microbial growth rates in milk mixtures and goat milk were comparable, with no significant increase in antibacterial activity due to donkey milk addition. Considering a potential employment of donkey milk in dairy products, further studies should be performed in order to detect the optimal balance of milk mixtures in terms of caseins and antimicrobial molecules amounts.
\end{abstract}

Keywords Donkey milk · Goat milk · Milk mixtures $\cdot$ Bacterial growth

Barbara Turchi

barbara.turchi@unipi.it

1 Dipartimento di Scienze Veterinarie, Università di Pisa, Viale delle Piagge 2, 56124 Pisa, Italy

2 Interdepartmental Research Center Nutrafood "Nutraceuticals and Food for Health", University of Pisa, Pisa, Italy 


\section{Introduction}

Consumers' interest in donkey milk started in the last two decades (Iacono et al. 1992; Carroccio et al. 2000) and rapidly increased, in relation to pediatric studies concerning cow's milk intolerance (Heine 2013; Sackesen et al. 2011).

Donkey milk is characterized by a remarkable content of antibacterial molecules, such as lactoferrin, which is two times higher than that of bovine milk (Malacarne et al. 2002) and lysozyme, which can reach levels of $4 \mathrm{mg} \cdot \mathrm{mL}^{-1}$, while only traces were found in bovine milk (Conte et al. 2012; Guo et al. 2007; Salimei et al. 2004). Consequently, donkey milk shows a natural low microbial content and an inhibitory effect against several microorganisms (Colavita et al. 2011; Conte et al. 2012; Sorrentino et al. 2010).

Currently, donkey milk is mostly purchased raw, pasteurized, or lyophilized, and its main employment is as alternative food for infants with cows' milk protein allergy. Some of the big issues for donkey milk market are represented by its high price, mostly due to the small amount of milk produced per day, a wavering consumers' demand, and the lack of a capillary distribution on the national market. These factors could sometimes contribute to make donkey milk difficult to be sold. In this context, transformation of donkey milk seems to be an important opportunity. If, on the one hand, donkey milk is unsuitable for cheese-making, due to its low caseins content, some studies have already pointed out an increased interest in the consumption of donkey milk in the form of fermented probiotic milks (Chiavari et al. 2005; Coppola et al. 2002). A fermented product could be also developed employing a mixture of two milks, such as donkey and goat milk: goat milk would benefit of an increased amount of nutraceutical compounds and antibacterial molecules; on the other hand, donkey milk would be enriched in caseins, with an improved organoleptic quality of the product. Moreover, goat milk, similarly to donkey milk, represents an alternative to cow milk for allergic consumers because of its lower $\alpha$-caseins content (Vincenzetti et al. 2014).

Galassi et al. (2012) and Cosentino et al. (2013) recently proposed the addition of donkey milk as an alternative to egg lysozyme in order to prevent blowing defect in Grana Padano cheese and ewe's cheese, respectively. According to Cosentino et al. (2013), the addition of $1.1 \% v / v$ donkey milk contributed to significantly reduce coliforms amount in cheese.

The aim of the present study was to preliminarily assess the survival growth rate of different pathogen microorganisms in milk mixtures consisting of goat milk and donkey milk in different percentages along 6 days of storage at refrigeration conditions $\left(4 \pm 2{ }^{\circ} \mathrm{C}\right)$ in order to evaluate whether the addition of donkey milk to goat milk could inhibit microorganisms replication.

\section{Materials and methods}

\subsection{Bacterial strains}

The type strains Staphylococcus aureus ATCC 6538, Listeria monocytogenes ATCC 7644TM, Pseudomonas aeruginosa ATCC 27853, and Campylobacter jejuni ATCC 33291 were employed for the tests. The strains were stored at $-80{ }^{\circ} \mathrm{C}$ in Brain Hearth 
Infusion broth (BHI) added with $20 \% v / v$ glycerol. Strains were revitalized on selective agar media at different optimal culture conditions. The media employed were Baird Parker agar (with egg yolk tellurite emulsion) at $37{ }^{\circ} \mathrm{C}$ for $24 \mathrm{~h}$ for $\mathrm{S}$. aureus; Agar Listeria Ottaviani-Agosti (with Listeria agar selective supplement and Listeria agar enrichment supplement) at $37^{\circ} \mathrm{C}$ for $24 \mathrm{~h}$ for L. monocytogenes; Pseudomonas CFC agar (Pseudomonas agar base with CFC supplement) at $30{ }^{\circ} \mathrm{C}$ for $24 \mathrm{~h}$ for P. aeruginosa and Campylobacter Blood-Free Selective Agar Base (with CCDA Selective Supplement) at $37^{\circ} \mathrm{C}$ in microaerophilic atmosphere for $24 \mathrm{~h}$ for $C$. jejuni. All media were purchased from Oxoid, (Milan, Italy), except for Agar Listeria Ottaviani-Agosti, which was purchased from Biolife Italiana s.r.l. (Milan, Italy).

\subsection{Donkey milk collection}

Donkey milk employed to detect antimicrobial activity against selected strains was collected from bulk-tank, where milk from several Romagnolo donkeys at different lactation stages was stored. For each tested bacterial strain a different donkey milk sample was used. Milk samples were collected immediately after milking and stored at refrigerated conditions during transportation to the laboratory. Donkey milk samples were treated at $68{ }^{\circ} \mathrm{C}$ for $10 \mathrm{~min}$ before their employment in the experiments.

\subsection{Donkey milk microbiological analysis}

Determination of total mesophilic bacterial count on Plate Count Agar (PCA) (Oxoid, Milan, Italy) was performed on treated donkey milk samples, before their employment in the trials. Enumeration was carried out after $72 \mathrm{~h}$ incubation at $30{ }^{\circ} \mathrm{C}$ in aerobic conditions. Moreover, the absence of each tested microorganism was verified.

\subsection{Lysozyme titration}

Whey was obtained from each treated donkey milk sample according to Carlsson et al. (1989) and stored at $-20^{\circ} \mathrm{C}$, until lysozyme titration, which was performed by lysoplate assay, according to Lie et al. (1986), with some modifications. Lysozyme (SigmaAldrich, Milan, Italy) dilutions ranging from $3 \mathrm{mg} \cdot \mathrm{mL}^{-1}$ to $0.003125 \mathrm{mg} \cdot \mathrm{mL}^{-1}$ were prepared in sterile saline and used as references. Our modification consisted in an incubation of the plates at $25{ }^{\circ} \mathrm{C}$ for $18 \mathrm{~h}$, which allowed a better evaluation of lysis halo diameters. After incubation, lysis halo diameters were measured and compared with those from lysozyme standard dilutions to obtain the lysozyme concentration of donkey milk samples used in our trials.

\subsection{Bacterial growth in milk mixtures}

For each strain, a bacterial suspension with a cellular concentration corresponding approximately to $1.5 \times 10^{7} \mathrm{CFU}_{\mathrm{mL}}^{-1}$ was prepared in sterile saline solution. Four UHT goat milk samples added with $1,2.5,5$, and $10 \%$ of donkey milk were then separately prepared. For each bacterial strain, $9 \mathrm{~mL}$ of each milk type were added with $1 \mathrm{~mL}$ of bacterial suspension. Two control samples, consisting of UHT goat milk and donkey 
milk inoculated with the standardized bacterial suspension, were also prepared. All samples were then stored at refrigerated conditions $\left(4 \pm 2{ }^{\circ} \mathrm{C}\right)$ for 6 days.

At inoculation time and after 1, 3, and 6 days of storage (T0, T1, T3, and T6, respectively), milk samples were serially diluted and grown on the agar media previously listed to enumerate bacterial cells. Bacterial growth was evaluated in milk mixtures, goat milk, and donkey milk. Each experiment was carried out in triplicate.

\subsection{Statistical analysis}

Statistical analysis was performed using the R v. 3.0.2 software ( $\mathrm{R}$ Foundation for Statistical Computing, Vienna, Austria). For each bacterial strain, the statistical significance of the differences among different types of milk was tested with a one-way ANOVA test followed by Tukey HSD post-hoc comparisons. Results were considered significant if associated with a $p$ value lower than 0.05 .

\section{Results and discussion}

\subsection{Donkey milk microbiological analysis}

All donkey milk samples employed in our trials showed a total bacterial mesophilic count lower than 100 CFU.mL ${ }^{-1}$. Moreover, Staphylococcus spp., Listeria spp., Pseudomonas spp., and Campylobacter spp. were not detected.

\subsection{Lysozyme titration}

Lysozyme concentration in donkey milk samples ranged from 1 up to $2 \mathrm{mg} \cdot \mathrm{mL}^{-1}$. In particular, donkey milk sample employed for S. aureus ATCC 6538 and L. monocytogenes ATCC 7644TM tests showed a lysozyme concentration of $1 \mathrm{mg} \cdot \mathrm{mL}^{-1}$, while donkey milk sample employed for P. aeruginosa ATCC 27853 and $C$. jejuni ATCC 33291 showed a lysozyme concentration of $2 \mathrm{mg} \cdot \mathrm{mL}^{-1}$.

\subsection{Bacterial growth in milk mixtures}

Table 1 shows microbial growth rates in milk mixtures and control samples.

As concerns $S$. aureus ATCC 6538, P. aeruginosa ATCC 27853, and C. jejuni ATCC 33291, bacterial growth rates in milk mixtures, donkey milk, and goat milk did not show any significant difference during the 6 days on refrigeration storage.

L. monocytogenes ATCC $7644 \mathrm{TM}$ growth rate showed instead a significant $(p<0.01)$ decrease in donkey milk starting from T1 compared to the other milks. Donkey milk revealed indeed an inhibitory effect against $L$. monocytogenes, with a decrease of 2.9, 2.51, and 5.55 $\log$ CFU.mL $\mathrm{mL}^{-1}$ at T1, T3, and T6, respectively. As concerns milk mixtures and goat milk, L. monocytogenes growth rate trend did not show significant differences.

During the storage from T0 to T6, an inhibited growth rate was also observed in donkey milk samples for $S$. aureus and $C$. jejuni, with a growth decrease of 0.61 and $1.72 \log$ CFU.mL ${ }^{-1}$, respectively. 


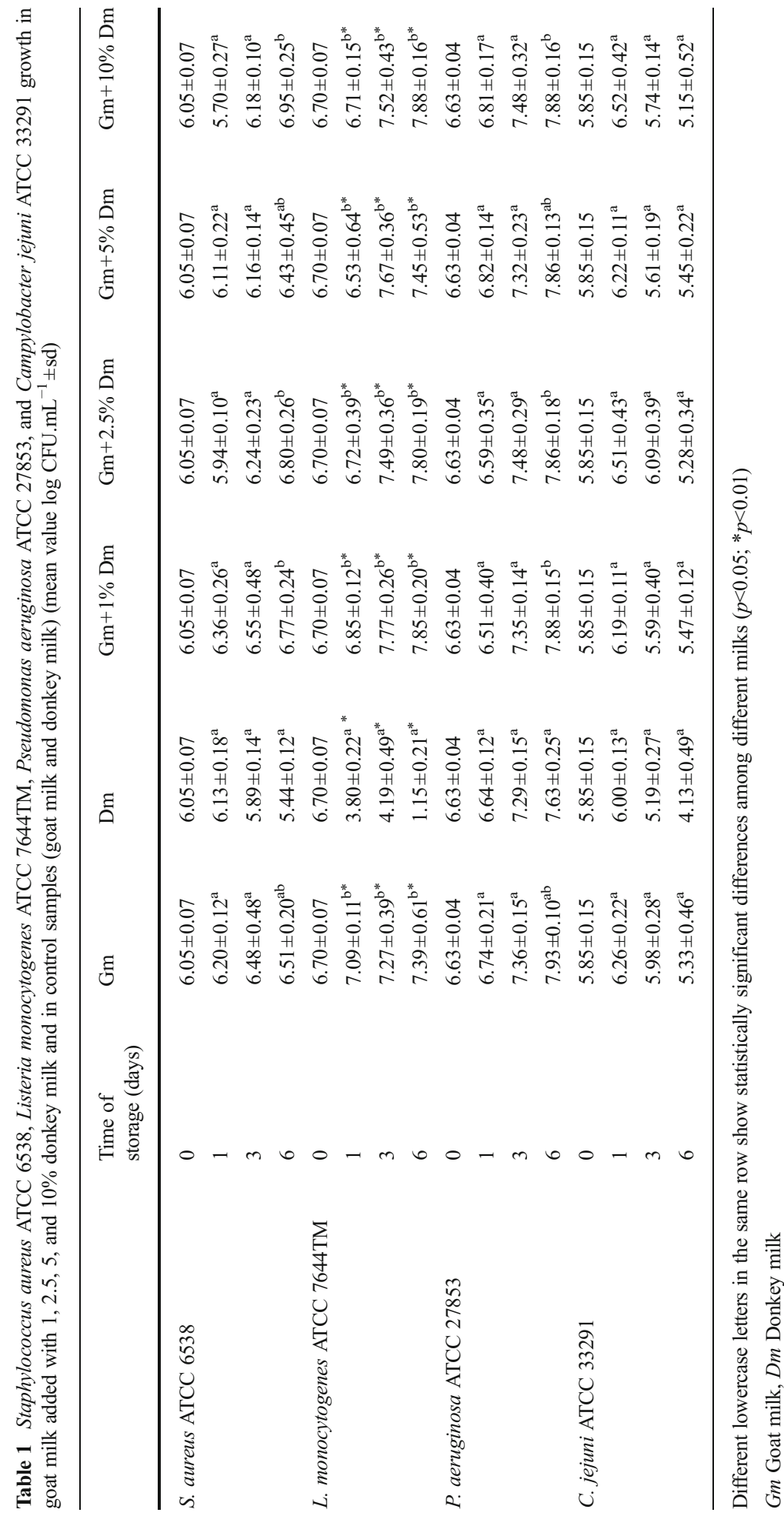


This work represents the first report on the evaluation of microorganisms' survival in goat and donkey milk mixtures. Lysozyme concentration found in donkey milk samples was in accordance with the recent literature, reporting an average lysozyme content ranging from 1 to $4 \mathrm{mg} \cdot \mathrm{mL}^{-1}$ (Coppola et al. 2002; Salimei et al. 2004; Zhang et al. 2008). It is important to highlight that donkey milk lysozyme content could vary not only in relation to the lactation period (D'Alessandro et al. 2011; Šrić et al. 2014a, b) but also to the analytical method (Chiavari et al. 2005; Coppola et al. 2002; Fantuz et al. 2001; Salimei et al. 2004; Vincenzetti et al. 2012). For our trials, donkey milk percentages $(1,2.5,5,10 \% \mathrm{v} /$ $v$ ) added to goat milk were chosen considering an average lysozyme content of $2 \mathrm{mg} \cdot \mathrm{mL}^{-1}$ and focusing on data obtained by Cosentino et al. (2013), who employed donkey milk in cheese-making to prevent blowing defects.

In the present work, microbial growth in goat milk was not affected by the additional amount of lysozyme provided by donkey milk. This amount ranged from 0.01 to $0.1 \mathrm{mg} \cdot \mathrm{mL}^{-1}$ for $S$. aureus and L. monocytogenes trials and from 0.02 to $0.2 \mathrm{mg} . \mathrm{mL}^{-1}$ for $P$. aeruginosa and $C$. jejuni trials. Thus, even though added lysozyme concentrations were equal or higher than those authorized by the manufacture regulation of some Italian cheeses, such as Grana Padano $\left(0.025 \mathrm{mg} \cdot \mathrm{mL}^{-1}\right)$, they were not effective in preventing the replication of inoculated microorganisms in milk mixtures. Besides, tested microorganisms were added in milk in a relatively high concentration (approximately $1.5 \times 10^{7} \mathrm{CFU} \cdot \mathrm{mL}^{-1}$ ) and this could have also contributed to the lack of inhibitory activity.

Another relevant aspect to be considered is the lysozyme activity at refrigeration temperatures; while Mariani (2010) has reported a lysozyme inactivity below $15{ }^{\circ} \mathrm{C}$, Šrić et al. (2012) have observed a donkey milk antimicrobial activity at temperatures equal and lower than $15^{\circ} \mathrm{C}$, also suggesting, as a possible explanation, a lysozyme nonenzymic mode of action (Šarić et al. 2014a, b). Our results seem to confirm this hypothesis, since in donkey milk samples, we observed a significant reduction of microbial growth.

The main results in terms of microbial inhibition were obtained for L. monocytogenes ATCC 7644TM growth in donkey milk. These results are noteworthy, especially taking into account the psychrophilic nature of L. monocytogenes and the high inocula concentration employed in the experiments. Indeed it is well known that lysozyme antimicrobial activity is mainly effective towards Gram-positive bacteria since peptidoglycan is freely accessible to the enzyme, while lipopolysaccharidic outer membrane layer shields Gram-negative cell wall (Bera et al. 2007; Masschalck et al. 2002; Veiga et al. 2006; Vollmer and Tomasz 2000). Our data are in accordance with Tidona et al. (2011), who have highlighted a significant antibacterial activity of digested and undigested donkeys' milk against L. monocytogenes (2230/92) in a dose dependent way.

\section{Conclusions}

Our results confirm donkey milk antimicrobial activity against some Gram-positive and Gram-negative strains (S. aureus ATCC 6538 and L. monocytogenes ATCC 7644 TM, C. jejuni ATCC 33291). Donkey milk percentages employed in our trials were not 
effective in inhibiting the development of tested microorganisms in milk mixtures. Further studies could be performed in order to detect the minimum donkey milk percentage able to provide an effective antibacterial activity when added to goat milk. Although this addition could represent a technological limit in a cheese-making process, due to a "dilution effect" towards caseins content, in the production of fermented milks it would not necessarily represent a challenging issue. The main beneficial aspects of further studies would be the opportunity to process donkey milk and make it less perishable. At the same time, donkey milk could represent an alternative lysozyme source to chicken egg-white lysozyme, often involved in allergic reactions in susceptible subjects due to its content in ovomucoid, ovoalbumin, and conalbuminin (Fremont et al. 1997; Mine and Zhang 2002).

Acknowledgments This work was supported by NU.FRO.LAS (NUove FROntiere per il Latte d'ASina) funds, issued within the 124 measure (Tuscany Region, Italy) for the rural development plan 2007/2013. The authors also would like to thank the Agriculture Cooperative Society "Extravaganti" for its encouragement and support.

Conflict of interest The authors declare that they have no competing interests.

\section{References}

Bera A, Biswas R, Herbert S, Kulauzovic E, Weidenmaier C, Peschel A, Götz F (2007) Influence of wall teichoic acid on lysozyme resistance in Staphylococcus aureus. J Bacteriol 189:280-283

Carlsson Å, Björck L, Persson K (1989) Lactoferrin and lysozyme in milk during acute mastitis and their inhibitory effect in Delvotest P. J Dairy Sci 72(12):3166-3175

Carroccio A, Cavataio F, Montalto G, D'amico D, Alabrese L, Iacono G (2000) Intolerance to hydrolysed cow's milk proteins in infants: clinical characteristics and dietary treatment. Clin Exp Allergy 30(11): 1598-1603

Chiavari C, Coloretti F, Nanni M, Sorrentino E, Grazia L (2005) Use of donkey's milk for a fermented beverage with lactobacilli. Lait 85:481-490

Colavita G, Amadoro C, Salimei E (2011) Latte di asina: aspetti igienico-sanitari e normativi. Argomenti SIVEMP 3:61-70

Conte F, Foti M, Malvisi M, Giacopello C, Piccinini R (2012) Assessment of antibacterial activity of donkey milk lysozyme. Safety and hygiene issues. Large Anim Rev 18(1):13-16

Coppola R, Salimei E, Succi M, Sorrentino E, Nanni M, Ranieri P, Belli Blanes R, Grazia L (2002) Behaviour of Lactobacillus rhamnosus strains in ass's milk. Ann Microbiol 52:55-60

Cosentino C, Paolino R, Freschi P, Calluso AM (2013) Short communication: Jenny milk as an inhibitor of late blowing in cheese: a preliminary report. J Dairy Sci 96:3547-3550

D'Alessandro AG, Martemucci G, Jirillo E, Leo VD (2011) Major whey proteins in donkey's milk: effect of season and lactation stage. Implications for potential dietary interventions in human diseases. Immunopharmacol Immunotoxicol 33(2):259-265

Fantuz F, Vincenzetti S, Polidori P, Vita A, Polidori F, Salimei E (2001) Study on the protein fractions of donkey milk. Proceedings of 14th Congress ASPA (Associazione Scientifica di Produzioni Animali), Firenze, Italy, 635-637

Fremont S, Kanny G, Nicolas JP, Moneret-Vautrin DA (1997) Prevalence of lysozyme sensitization in an eggallergic population. Allergy 52(2):224-228

Galassi L, Salimei E, Zanazzi M (2012) Impiego del latte di asina in sostituzione di lisozima da uovo nella produzione del formaggio duro italiano: prime esperienze [Grana Padano cheese making with lysozyme from ass's milk: first results]. J Ital Dairy Sci Assoc (AITeL) 63(1):73-79

Guo HY, Pang K, Zhang XY, Zhao L, Chen SW, Dong ML, Ren FZ (2007) Composition, physiochemical properties, nitrogen fraction distribution, and amino acid profile of donkey milk. J Dairy Sci 90:16351643

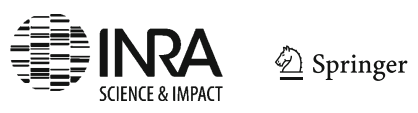


Heine RG (2013) Cow's-milk allergy and lactose malabsorption in infants with colic. J Pediatr Gastroenterol Nutr 57:S25-S27

Iacono G, Carroccio A, Cavataio F, Montalto G, Soresi M, Balsamo V (1992) Use of ass' milk in multiple food allergy. J Pediatr Gastroenterol Nutr 14(2):177-181

Lie Ø, Syed M, Solbu H (1986) Improved agar plate assay of bovine lysozyme and haemolytic complement activity. Acta Vet Scand 27:23-32

Malacarne M, Martuzzi F, Summer A, Mariani P (2002) Protein and fat composition of mare's milk: some nutritional remarks with reference to human and cow's milk. Int Dairy J 12:869-877

Mariani PL (2010) Donkey milk nutraceutical characteristics: a biochemical evaluation of nutritious and clinical properties. http://cameprints.unicam.it/284/1/Donkey_milk.pdf Accessed 25 June 2015

Masschalck B, Deckers D, Michiels CW (2002) Lytic and nonlytic mechanism of inactivation of grampositive bacteria by lysozyme under atmospheric and high hydrostatic pressure. J Food Prot 65:19161923

Mine Y, Zhang JW (2002) Comparative studies on antigenicity and allergenicity of native and denatured egg white proteins. J Agric Food Chem 50:2679-2683

Sackesen C, Assa'ad A, Baena-Cagnani C, Ebisawa M, Fiocchi A, Heine RG, Von Berg A, Kalayci O (2011) Cow's milk allergy as a global challenge. Curr Opin Allergy Clin Immunol 11(3):243-248

Salimei E, Fantuz F, Coppola R, Chiofalo B, Polidori P, Varisco G (2004) Composition and characteristics of ass's milk. Anim Res EDP Sci 53(1):67-78

Šarić LĆ, Šarić BM, Mandić AI, Torbica AM, Tomić JM, Cvetković DD, Okanović ĐG (2012) Antibacterial properties of domestic Balkan donkeys' milk. Int Dairy J 25(2):142-146

Šarić LĆ, Šarić BM, Mandic AI, Tomic J, Torbica A, Nedeljkovic N, Ikonic BB (2014a) Antibacterial activity of donkey milk against Salmonella. Agro Food Ind Hi Tech 25:5

Šarić LĆ, Šarić BM, Mandić AI, Kevrešan ŽS, Ikonić BB, Kravić SŽ, Jambrec DJ (2014b) Role of calcium content in antibacterial activity of donkeys' milk toward E. coli. Eur Food Res Technol 239(6):1031-1039

Sorrentino E, Di Renzo T, Succi M, Reale A, Tremonte P, Coppola R, Salimei E, Colavita G (2010) Microbiological characteristics of raw ass's milk: manual vs. machine milking. 61st annual meeting of the European Association for Animal Production, Wageningen, The Netherlands:Wageningen Academic Publishers, Book of Abstract 16:44

Tidona F, Sekse C, Criscione A, Jacobsen M, Bordonaro S, Marletta D, Vegarud GE (2011) Antimicrobial effect of donkeys' milk digested in vitro with human gastrointestinal enzymes. Int Dairy J 21(3):158-165

Veiga P, Piquet S, Maisons A, Furlan S, Courtin P, Chapot-Chartier MP, Kulakauskas S (2006) Identifcation of an essential gene responsible for DAsp incorporation in the Lactococcus lactis peptidoglycan crossbridge. Mol Microbiol 62:1713-1724

Vincenzetti S, Amici A, Pucciarelli S, Vita A, Micozzi D, Carpi FM, Polzonetti V, Natalini P, Polidori P (2012) A proteomic study on donkey milk. Biochem Anal Biochem 1:109

Vincenzetti S, Foghini L, Pucciarelli S, Polzonetti V, Cammertoni N, Beghelli D, Polidori P (2014) Hypoallergenic properties of donkey's milk: a preliminary study. Vet Ital 50(2):99-107

Vollmer W, Tomasz A (2000) The pgdA gene encodes for a peptidoglycan N-Acetylglucosamine deacetylase in Streptococcus pneumonia. J Biol Chem 275:20496-20501

Zhang XY, Zhao L, Jiang L, Dong M, Ren F (2008) The antimicrobial activity of donkey milk and its microflora changes during storage. Food Control 19:1191-1195 